\title{
Detection And Face Recognition of People BY CAMERA FOR SECURITY APPLICATIONS
}

\author{
Barbora Kotkova, Martin Hromada, Vaclav Mach \& Marta Blahova
}
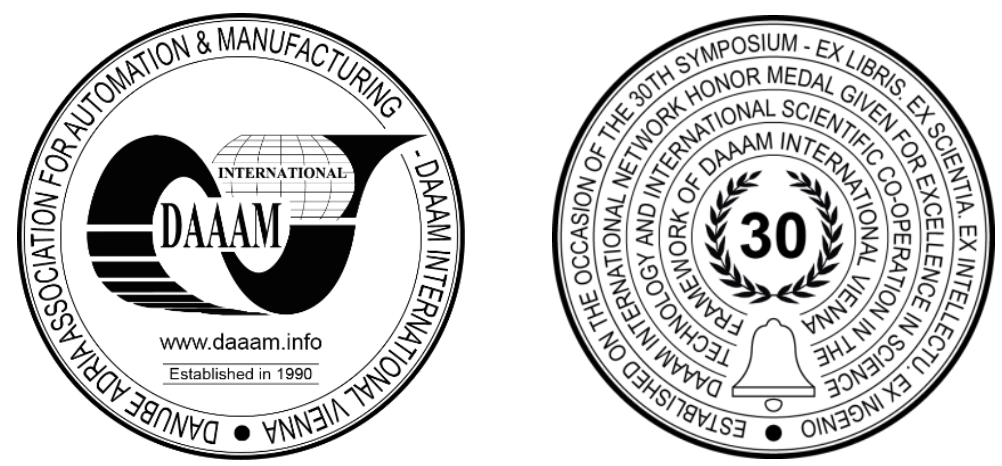

This Publication has to be referred as: Kotkova, B[arbora]; Hromada, M[artin]; Mach, V[aclav] \& Blahova, M[arta] (2019). Detection and Face Recognition of People by Camera for Security Applications, Proceedings of the 30th DAAAM International Symposium, pp.0610-0614, B. Katalinic (Ed.), Published by DAAAM International, ISBN 978-3-90273422-8, ISSN 1726-9679, Vienna, Austria

DOI: $10.2507 / 30$ th.daaam.proceedings.083

\begin{abstract}
This article discusses the possibility of applying software evaluation for detection and recognition in the inside and outside security areas. The methods of face detection and recognition, the methods used in practice are listed in the description. The program Wolfram Mathematica software was used for face detection with a subsequent comparison with the database of persons and outlines methods of testing according to the international standard. Finally, the testing of these software programs is described with a focus on their reliability according to predetermined scenarios. There are listed the final comparison between several evaluation programs which can process the face detection.
\end{abstract}

Keywords: Camera; Face detection; Identification of people; Software; Wolfram Mathematica.

\section{Introduction}

Recognizing individual objects, things, people and perceptions are one of the basic human abilities. The human senses are adapted to allow one to orient and make the right decisions. In fact, through vision, we acquire new information or verify information already obtained. The people create various important databases in memory that are interconnected with each other and which are constantly filled with new data and compared with the data already stored.

Around 1970, digital image processing took place, and in the years to come, scientists gradually deal with the 3D structure of objects. Gradually, the area develops in multiple directions, one of which has been object detection and person detection. [1] Detecting people using a visual manner is very popular because it can provide a huge application in many fields. The facial detection is mainly used in the stationary cameras which are mounted next to the entrance to scan people. The camera sent the image to the evaluation program. The program has access to a huge database with different people. The program finds facial landmarks and compares them with the database.

According to the author [2] the problem of finding and analyzing faces is a foundational task in computer vision. Though great strides have been made in face detection, it is still challenging to obtain reliable estimates of head pose and facial landmarks, particularly in unconstrained images. This method uses a contactless process, so it becomes popular. 
According to the [3] facial landmarks or facial fiducial points have semantic meaning. Facial feature points are mainly located around facial components such as eyes, mouth, nose, and chin. Author [3] also claims that facial feature points can be reduced to three types: points labeling parts of faces with application dependent significance, such as the center of an eye or the sharp corners of a boundary; points labeling application-independent elements, such as the highest point on a face in a particular orientation, or curvature extrema (the highest point along the bridge of the nose); and points interpolated from points of the previous two types, such as points along the chin. According to this information, an $n-$ points model can be created.

\subsection{Possibilities of using data from the camera system}

Social perception of monitoring people and collecting personal information is a very sensitive topic. Most residents do not want their photographs or biometric data to be stored in the databases of different companies, and therefore it is necessary to ensure that the legal conditions regarding personal data are observed. [1] In commercial areas, the FR is already being used to identify persons at the perimeter level in terms of whether an unauthorized person is trying to penetrate the object of interest. If such a situation is identified, there is sufficient time for operators to respond adequately to the potential risk of misuse of information or damage to company assets. [1]

A typical case is when an unauthorized person enters the area. Using the CCTV, images of a person are obtained, which are then sent to a server with installed Face Recognition software, which performs a comparison and, if the person is placed on a "Black List", sends this information to the security system operator. [1] Some incidents do not, by their severity and scope, meet the parameters for taking action by the Public Order and Security System, but for the soft objective alone, they are so seriously interfering with a routine that it is appropriate to apply procedures for major security incidents. [4]

The next category is the use of Files in the area of security. The security situation is deteriorating from the point of view of terrorism and extremism in Europe, and there is an increase in terrorism-like, not ideologically motivated, violent attacks, just like the terrorist ones, on soft targets in order to hurt accidentally present persons. [4] However, the area of personal identification is also widely used by police authorities, intelligence services or public authorities. The Police of the Czech Republic has the legal opportunity to monitor public space, which is also actively used and in each police vehicle is integrated at least two cameras, which continuously record. [1]

\subsection{Procedure to Identify the Person}

Before we begin to identify people, we need to solve the problem of their detection in the video. It is not only necessary to detect movement, but also to determine the exact position of a person and to determine which pixels match them. [5] The identification process is based on these segments:

- $\quad$ Pre-processing - image cleansing from optical errors and noise

- Segmentation - dividing the image into individual segments, assigning the index number segment. It separates important parts of the image from the unimportant ones. Methods - edge detection, neural networks, threshholding and more.

- Object description - division of found objects into classes according to size, color, location, and shape

- Face detection - using methods (depending on image processing, shooting method) or algorithms, in practice also combinations thereof.

- Face comparison - based on the input data is searched for the identity of the person in the database, signing identity and evaluating the success

Problems in detecting people close together appear as one figure after background reading. Another problem may be the incorrect detection of one person. There could be a situation where, after subtracting the background, we see one person divided into multiple parts. When designing the system, it is appropriate to take into account the layout of the cameras. Improper detection of foreign objects could become an unpleasant thing. Phenomena such as opening and closing doors can be identified as a short movement at one point in the scene. It may happen that the system will try to identify the door as a person. This can be avoided either by searching for a person's role model for each part or by requiring some movement from the person. [5]

\subsection{Methods used for Detection and Identification}

There are many methods for detecting a face in an image. In this chapter, it is possible to get acquainted with theirs The following sub-chapters describe some of them. According to publication [6] of 2002, by Yang, Kriegman, and Ahuja, methods for face detection were divided into the following four basic groups [7] The first on is the Knowledge-based methods where the rules capture the relationships between typical facial features. The second is the feature invariant 
methods where the program looks for typical features that are independent of the angle of rotation of the head and light conditions. The next is the template matching methods where face detection is based on the correlation between the input image and the pattern face or parts of it. The last is the appearance-based methods where a model is created with this method sets containing a variety of possible facial expressions.

Methods used for face recognition can be structural - recognition of individual dominant parts of the face (eyes, mouth, nose...) of the presented pattern, measuring anthropometric quantities, their normalization due to expected interference (noise, interference, position in the scene, size...), comparison with a database of known photos using classification algorithms, statistical decisions about the relative similarities with the selected set of images. The second can be holistic - comparison, sample identification using global representations again with a subsequent statistical evaluation of the relative probability. Typical for this approach are combinations of the backpropagation method neural network feedback), basic component analysis (PCA) and singular value decomposition (SVD). The concept of reductionism is a general practice in the development of intelligent systems - design of solutions to complex problems through the gradual decomposition of the task into subsequent modules. Addressing the task of identifying stakeholders can be a combination of both. [8]

\section{Experimental section}

There are many products on the market with different algorithms. It is not unusual that two different software manufacturers use the same algorithm with different set parameters. To measure the performance and quality of facial recognition algorithms, the US National Institute for Standards and Technology (NIST) has introduced methodologies that test the capabilities of software from different vendors. [9]

\subsection{Program for face detection in Wolfram Mathematica}

The author [10] claims that one of the elements in building this experiment is a Wolfram Language built-in function: FindFace. This function is still in the improvement phase and has several issues, from showing non-face examples and having a problem with rotated pictures. Even in dynamic mode, all processes are mapped into many single frames; so some wrong results may be shown when the picture is changing rapidly. Each face must be first identified by the program. Wolfram Mathematica has a built-in function which automatically selects all faces. The input to this function can be dynamic video, or it can be the image. The output of this function is the same image with the highlighted rectangle. Wolfram Mathematica uses many online libraries which provides a lot of function that is free to use.

The captured image by the camera is sent via the video transmitting device to the USB converter. Tested people were in the classroom with a lot of obstacles. The face detection is done using the following code, and the graphical output is in the following figure where all faces are highlighted by the red rectangle. The face detection is done using the following code, and the graphical output is below.

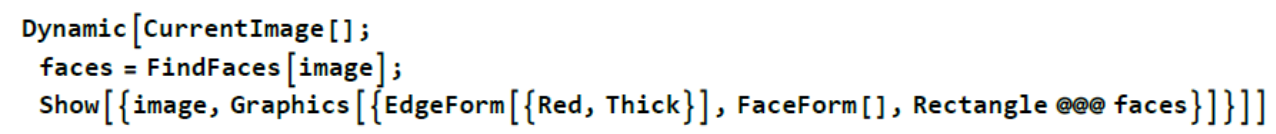

Fig. 1. Function for the finding faces

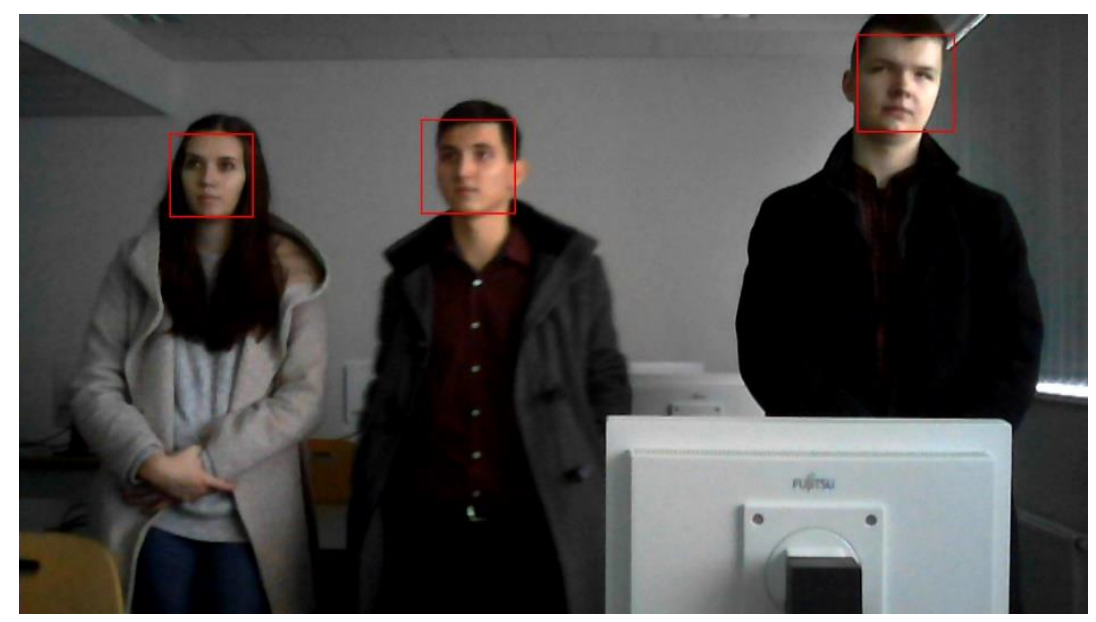

Fig. 2. Input image with highlighted faces

To reduce the amount of information the next function is used in the program. This function cut out the highlighted faces. The code and the output can be seen in the following figures. 


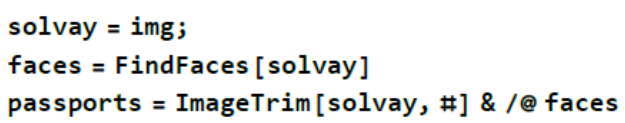

Fig. 3. Function for cutting faces
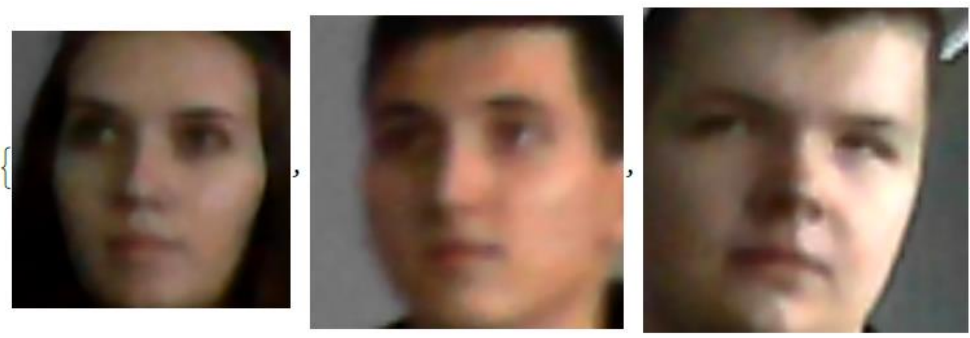

Fig. 4. Image with the cut faces

To identify the face from the image program needs to find some specific points on the face. Wolfram Mathematica has a function for corner identification. The face does not have any corners; however, this function fits very well in this situation and it is commonly used for face identification. The code and the output can be seen in the following figures.

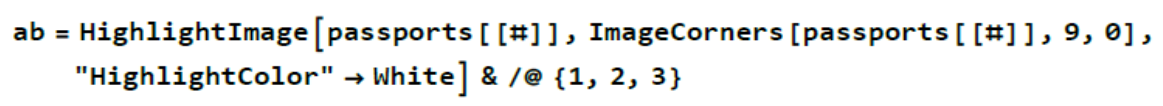

Fig. 5. Function for finding corners
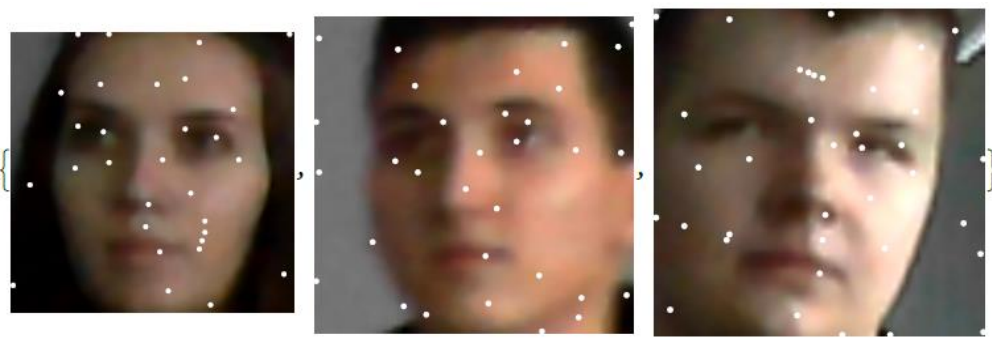

Fig. 6. Image with marked corners on the faces

The program finds the corners in the bit map, and these dots can be then compared with others. One of the advantages of this method is that the program can identify face even if it is taken from a different angle or mirrored.

\section{Comparison of selected Software}

There are many types of software that have different procedures and outputs. However, the conclusion must always be the same - the person being compared is located / not in the database. As an example of data processing and its outputs, user testing of two software from Japanese company NEC Corporation and IsVision company AnyVision. The purpose of the testing was not to verify all the declared functions, but the testing was aimed primarily at verifying the reliability of detection and identification of persons.

For testing was used a database, which was used about 13000 Mugshots type. The database includes men and women aged 15-80 years and of different ethnicities, with men and women of Caucasian origin being the most represented. However, some photographs were intentionally taken in situations where it was already difficult for a person to determine whether they were indeed the person to be found in the default database. Both products had their own friendly user interface. First, you had to connect to the default database (a database of 13,000 photos). Subsequently, a reference photo of the person we were looking for was uploaded to the default database.

The testing focused on different types of environments and situations. In the first test, the systems were to detect a seated man at the age of 23 , and the following test set was aimed at a white woman. In addition, a black man of approximately 50-55 years was selected. The last test set was aimed at a woman with Asian roots. Person detection and identification for Better Tomorrow and Neo Face Watch are only part of the data tested. the input images were intentionally taken in reduced quality or in situations where the wanted persons are in different positions on the border of the declared algorithm possibilities. 


\subsection{Results of the testing}

With Neo Face Watch, 38\% of the images tested were correctly identified in a predetermined scenario. Better Tomorrow's success rate was 53\%. Although Better Tomorrow had a lower match than Neo Face Watch throughout the testing, it was able to correctly assign an identity to multiple people. It follows that the score cannot be taken as a decisive parameter for determining which algorithm to be tested is more reliable.

The Neo Face Watch software has always suggested several candidates that match the person on the embedded image. The searched person was, in the vast majority of cases, nominated among the top five candidates, and most often it was the first candidate, the highest probability of being the same person as the reference image.

Using the Wolfram Mathematica there was no opportunity to see other candidates with a lower match, and only one candidate was introduced to the tester, but it was $99 \%$ correct. Both manufacturers declare in their marketing materials a rate of correct identification of greater than $99 \%$ and a misidentified person of less than one $1 \%$ per day. From the test results, it can be concluded that the declared value manufacturers are achievable provided the requirements for image quality and visibility of the major minutiae of the face are respected.

\section{Conclusion}

The main goal of this article was to compare the current software which is focused on face detection. The Wolfram Mathematica software was used for comparison. During the testing, it was found that if the algorithm can correctly detect a person's face in the image, the subsequent identification against the reference image is at a very high level. These results were obtained based on a single image that was embedded in the software. CCTV systems are used to monitor areas in the security area. Current cameras capture the scene in high definition and the frame rate has increased. In the case of installing a CCTV system and simultaneously identifying software, people acquire much higher quality input photos than if a single photo is used for comparison.

The results will be affected by whether the images are taken indoors or outdoors. The comparison showed that the used software is very effective and flawless. A high-resolution camera, outdoor, low visibility, and poor weather conditions do not necessarily guarantee an increased likelihood of identifying the person. Another aspect that will affect the image quality of detected people is whether the images will be obtained from a collaborative or non-cooperative scene. Further research can be focused on processing the video signal by the Wolfram Mathematica instead of the stable image.

\section{Acknowledgments}

This work was supported by the Ministry of Education, Youth and Sports of the Czech Republic within the National Sustainability Program Project No. LO1303 (MSMT-7778/2014) and also by the European Regional Development Fund under the project CEBIA-Tech ED2.1.00/03.0089 and by the Internal Grant Agency of Tomas Bata University under the project No. IGA/CebiaTech/2019/003 and the IGA/FAI/2019/003.

\section{References}

[1] Gabko, L. (2018). Testování spolehlivosti software určeného pro detekci osob. Available from: http://hdl.handle.net/10563/44437.

[2] Xiangxin Z. \& Ramanan, D. (2012) Face detection, pose estimation, and landmark localization in the wild. Conference on Computer Vision and Pattern Recognition. pp. 2879-2886. DOI: 10.1109/CVPR.2012.6248014. ISBN 978-1-4673-1228-8

[3] Wang, N., Gao, X., Tao, D., Yang, H. \& Li, X. (2018) Facial feature point detection: A comprehensive survey. Neurocomputing. pp. 50-65. DOI: 10.1016/j.neucom.2017.05.013. ISSN 09252312.

[4] Lapkova, D., Kotek, L. \& Kralik L. (2018). Soft Targets - Possibilities Of Their Identification. Katalinic, Branko, ed. Proceedings of the 29th International DAAAM Symposium 2018. DAAAM International Vienna, pp. 0369-03. DOI: 10.2507/29th.daaam.proceedings.053. ISBN 9783902734204.

[5] Hopjan, T. (2010). Identifikace osob pro kamerový systém. Available from: http://hdl.handle.net/11012/53000.

[6] Ming-Hsuan, Y., Kriegman D.J. \& Ahuja N. (2002). Detecting faces in images: a survey. IEEE Transactions on Pattern Analysis and Machine Intelligence. vol. 24, issue 1, pp. 34-58. DOI: 10.1109/34.982883.

[7] Jablonski, P., Szewczyk R., Kulesza Z., Napieralski A., Moreno M. \& Cabestany J. (2002) Automatic people identification on the basis of iris pattern - image processing and preliminary analysis. 23rd International Conference on Microelectronics. pp. 687-690. DOI: 10.1109/MIEL.2002.1003351. ISBN 0-7803-7235-2.

[8] Ponzer, M. (2009). Detekce a rozpoznávání obličeje. Available from: http://hdl.handle.net/11012/2006.

[9] Wu, W., Yin Y., Wang, X. \& Xu D. (2019). Face Detection with Different Scales Based on Faster R-CNN. IEEE

[10] Alionte, E. \& Lazar, C. (2015) A practical implementation of face detection by using Matlab cascade object detector. 19th International Conference on System Theory, Control and Computing (ICSTCC). pp. 785-790. DOI: 10.1109/ICSTCC. 2015.7321390. ISBN 978-1-4799-8481-7. 